\title{
A large, searchable, web-based database of aphasic performance on picture naming and other tests of cognitive function
}

\author{
Daniel Mirman \\ Moss Rehabilitation Research Institute Elkins Park, PA 19027 \\ Ted J. Strauss \\ New School for Social Research New York, NY 10003 \\ Adelyn Brecher \\ Moss Rehabilitation Research Institute Elkins Park, PA 19027 \\ Grant M. Walker \\ Department of Neurology, University of Pennsylvania Philadelphia, PA 19103 \\ Paula Sobel \\ Moss Rehabilitation Research Institute Elkins Park, PA 19027 \\ Gary S. Dell \\ Beckman Institute, University of Illinois Urbana, IL 61801 \\ Myrna F. Schwartz ${ }^{*}$ \\ Moss Rehabilitation Research Institute Elkins Park, PA 19027
}

\begin{abstract}
Many research questions in aphasia can only be answered through access to substantial numbers of patients and to their responses on individual test items. Since such data are often unavailable to individual researchers and institutions, we have developed and made available the Moss Aphasia Psycholinguistics Project Database: a large, searchable, web-based database of patient performance on psycholinguistic and neuropsychological tests. The database contains data from over 170 patients covering a wide range of aphasia subtypes and severity, some of whom were tested multiple times. The core of the archive consists of a detailed record of individual-trial performance on the Philadelphia (picture) Naming Test. The database also contains basic demographic information about the patients and patients' overall performance on neuropsychological assessments as well as tests of speech perception, semantics, short-term memory, and sentence comprehension. The database is available at http://www.mappd.org/.
\end{abstract}

\section{Keywords}

aphasia; database; picture naming; language

Cognitive neuropsychological studies rely on three core methods: in-depth studies of single cases, comparisons of two or more patients or patient groups that demonstrate dissociations between cognitive functions, and case series that evaluate patterns of performance over a large series of patients. Although all of these methods have provided important insights

\footnotetext{
"Corresponding author: Myrna F. Schwartz Moss Rehabilitation Research Institute 50 Township Line Rd., Elkins Park, PA 191027, USA (215) 663-6036 (T), (215) 663-6783 (F) mschwart@ einstein.edu.
} 
about cognition and its neural basis, case series studies, in particular, have been increasingly common in the field. Proponents of this method claim that it is particularly well suited to establishing the validity of theoretically-relevant symptom associations, as well as dissociations (Schwartz \& Dell, 2010; Patterson \& Plaut, 2009).

The promise of the case series method for theory development is countered by the difficulty of collecting such data. The primary difficulty, of course, is that of recruiting targeted patients on a large scale. Even in facilities that treat patients with cognitive impairments, suitable candidates are sparsely represented in any single facility; and federal and institutional privacy protections pose significant barriers to identifying and contacting patients once they leave the system. Models exist for how to build a suitable recruitment infrastructure for cognitive neuroscience and rehabilitation research (Schwartz, Brecher, Whyte, \& Klein, 2005). Considerable resources, however, are required to maintain such an infrastructure and to move patients through the steps of consenting, screening, and testing. One can assume that for the foreseeable future, large-scale patient recruitment and testing will remain impossible for most individual researchers and institutions. Data sharing provides a solution to this problem. To that end, we have developed and made available the Moss Aphasia Psycholinguistics Project Database: a large, searchable, web-based database of patient performance on the Philadelphia (picture) Naming Test (PNT; Roach et al., 1996) and a wide array of ancillary psycholinguistic and neuropsychological tests. The database is available at http://www.mappd.org/.

\section{Database Content}

\section{Patients}

The web database represents years of data collection (starting in 1991 and still ongoing) from patients who exhibited language impairments secondary to chronic left hemisphere stroke. Most were recruited to the research program at Moss Rehabilitation Research Institute (MRRI) through an IRB-approved, consent-based recruitment infrastructure we call the Patient Registry. All gave voluntary consent to participate in the research program and to allow the anonymized results of this research to be published. Accordingly, the web database contains no individually identifying information about the patients. Individual patients are listed only by arbitrary codes (e.g., "MR00409"), with only relative time information provided (e.g., "months post onset" and "age at testing").

Patients in the web database were between the ages of $18-80$ and were mostly monolingual English speakers (less than 5\% reported speaking a second language and English was the first and primary language for all patients). The vast majority were chronic (> 6 mo. post stroke) and, by clinical criteria, some would be considered recovered (e.g., Western Aphasia Battery Aphasia Quotient (AQ)> 93.8). The broad inclusion criteria allowed for the collection of data from a large and diverse set of patients. Currently the database contains data from 178 patients (mostly Anomic, Broca's, Conduction, and Wernicke's subtypes) covering a wide range of severity (AQ: 33.3 - 97.8). Some were tested multiple times, allowing for examination of recovery patterns (at least 20 patients were tested 5 times). The database also contains basic demographic information (age, ethnicity, and years of education) and neurological information (months-post-onset, aphasia subtype, and apraxia of speech).

\section{The Philadelphia Naming Test}

The core of the database is individual-trial performance data from picture naming. Picture naming is a primary test of lexical processing. The task taps a critical juncture in the language system because naming mediates between high-level conceptual and syntactic processing and low-level phonological processing. Thus it is not at all surprising that 
difficulty in this task is present to varying degrees in nearly all aphasic individuals. The PNT specifically has featured in the development and evaluation of an influential model of lexical access in production (Dell et al., 1997; Schwartz et al., 2006). In the 15 years since its publication, the PNT has been used in many studies testing theories of production by investigating the distributions of aphasic picture naming errors (e.g., Dell et al., 1997; Dell, Lawler, Harris, \& Gordon, 2004; Dell, Martin, \& Schwartz, 2007; Foygel \& Dell, 2000; Kittredge, Dell, Verkuilen, \& Schwartz, 2008; N. Martin \& Dell, 2007; Schwartz et al., 2006; Nozari, Kittredge, Dell, \& Schwartz, 2010). Other studies using the PNT have examined conceptual representations (e.g., Buxbaum \& Saffran, 2002; Saffran, Coslett, \& Keener, 2003; Saffran, Coslett, Martin, \& Boronat, 2003), short-term memory (e.g., Martin \& Ayala, 2004; Freedman, Martin, \& Biegler, 2004), and the effects of treatments on recovery of language function (e.g., Fridriksson et al., 2007; Jokel, Rochon, \& Leonard, 2004; Leonard, Rochon, \& Laird, 2008; Schwartz \& Brecher, 2000)

The PNT is a 175-item single word picture-naming test developed for collecting a large corpus of naming responses from a standardized set of items. The pictured items were selected on the basis of their familiarity, name agreement, and good image quality (blackand-white line drawings of minimal complexity and confusability). Target names are all basic level concepts (i.e., not subordinate or superordinate; no targeting of famous faces or landmarks) and cover a relatively wide range of word length (1 to 4 syllables), word frequency (1 to 2110 tokens per million of printed English text; Francis \& Kucera, 1982), and semantic category (animals, body parts, clothing, food, furniture, tools, vehicles, etc.). Concept familiarity and name agreement are also reflected by the near-perfect (97\% correct) naming performance of unimpaired controls (Dell et al., 1997; Roach et al., 1996) and itemlevel responses from control participants are included in the database for researchers interested in common errors. The details of test administration and scoring are described elsewhere (Roach et al., 1996). A detailed scoring guide is provided in the documentation section of the database website along with a link to the PNT page

(http://mrri.org/index.php/materials-and-methods/philadelphia-naming-test), which contains all of the test materials, administration instructions, score sheets, scoring procedures etc. Basic psycholinguistic characteristics of each target word (lexical frequency, visual complexity, length, semantic category, etc.) are included in the web database.

The database contains trial-level data for the PNT and PRT (a word repetition test using the same set of 175 targets as the PNT). For each administration of each test, for each of the target items, the database contains a transcription (phonetic and orthographic) of the patient response (first complete attempt), and two different categorizations of the type of response. The first is a "conventional" response code that includes common response types (correct, semantic error, formal error, nonword, description, no-response, etc.) as well as less common response types (picture part, perseveration, abstruse neologism, etc.). The second is a narrower coding of response types that was developed in order to test the interactive twostep computational model of speech production (Dell et al., 1997; Dell \& O'Seaghdha, 1991; Foygel \& Dell, 2000). This "model" response coding scheme only includes six response types: correct, semantic error, formal error, mixed error, nonword, and unrelated. In the model coding scheme, responses that do not fit these categories are left blank so that averaging will produce the normalized error proportions used to evaluate the model (see Dell et al., 2004; Schwartz et al., 2006). For each administration of the PNT, the database also contains two sets of parameters derived from fitting the distribution of error types using the model. There are two sets of parameters because the model can be fit by altering weight strength and activation decay parameters (w-d model) or by altering semantic and phonological weight strengths (s-p model), for details see Foygel and Dell (2000) and Schwartz et al. (2006). Investigators wishing to apply the model to new data can access it at http://langprod.cogsci.uiuc.edu/cgi-bin/webfit.cgi. 


\section{Additional Tests}

In addition to the item-level PNT and PRT data, the database also contains patients' overall performance on a variety of ancillary psycholinguistic and neuropsychological tests. These tests were developed by different research labs and focus primarily on semantic and phonological word processing, short-term memory, and non-verbal (picture) semantics. There are no tests of written language processing or non-language neuropsychological functions in the database. Although the database has been used in studies of lesion-symptom mapping (e.g. Schwartz et al., 2009), at present, it does not contain lesion data. The complete set of tests is listed in Table 1 and brief descriptions of each test are provided in Table 2 (more details are available at the database website). The documentation section of the website includes the tests that we have permission to distribute with information for their use.

The web database will be updated periodically as data collection continues. Additional patients and test administrations will be added, so the patient and test descriptions provided here describe the initial database contents. Users should consult the online database documentation, which will contain the most current counts and descriptions.

\section{Database Functions}

This database is intended to spur basic and clinical research on the neuropsychology of language by providing easy access to results that can speak to a wide variety of research questions. Broadly, database queries can be formulated around three types of constraints: (1) patient characteristics such as aphasia subtype, education level, performance on short-term memory tests, etc., (2) test item characteristics such as word frequency, semantic category, etc., and (3) picture naming performance characteristics such formal errors, nonwords, descriptions, etc. In addition to query constraints, the web-based interface allows specification of which data should be provided in the output and simple averaging and sorting tools for organization of the output. The default output format is an easy-to-read table. Alternatively, the website can provide the output as a comma-separated-values simple text table that can be copied and read into standard data analysis and visualization software.

For example, a researcher may wish to examine whether the distribution of picture naming errors differs as a function of aphasia subtype (i.e., select the error types and aphasia subtypes of interest for output, and average by aphasia subtype), or to look for specific patterns within aphasic nonword errors (i.e., constrain the search to nonword errors and then select test word and phonetic and/or regular (orthographic) response for output), or to investigate whether error types differ between semantic categories such animals and vehicles (i.e., select the error types of interest and semantic category, and average by semantic category). These constraints can also be combined to answer more specific research questions: What kinds of descriptions are produced by patients who make many semantic errors? (Constrain patients to those who make a criterion number of semantic errors and responses to descriptions). In addition to the tremendous power of item-level picture naming data, the inclusion of other psycholinguistic tests in the database allows investigation of questions that are unrelated to picture naming. For example, how strong is the relationship between word recognition (i.e., lexical decision) and short-term memory? (Do not constrain the search, output Anonymous subject ID, lexical decision, and short-term memory scores, and finally average by subject ID). Detailed documentation and user's guide, including a step-by-step How-To guide for performing searches, are available on the database website.

A recent paper, Nozari et al. (2010), used the database to compare the influence of lexical frequency on aphasic naming and auditory word repetition. Most theories of word production associate frequency with the retrieval of phonological forms from an abstract 
lexical/semantic representation (e.g. Kittredge et al., 2008). This frequency-sensitive processing step is clearly involved in naming, but does it also play a role in word repetition? If repetition is routinely carried out by a non-lexical route in which processing goes directly from input to output phonology, this frequency-sensitive step is bypassed and, consequently, frequency effects should be much smaller in repetition than in naming. Alternately, word repetition could be mediated by the access of an abstract lexical representation that is then pronounced using the same frequency-sensitive step that is involved in naming. Because the database contains both naming and repetition errors from the same items and patients, this question can be easily answered using its features. Specifically, Nozari et al. assessed nonword-error probability as a function of task (naming vs. repetition) and the lexical properties of the target item, which were obtained from the database (frequency, length, phonological density, imageability, name agreement). Only trials from patients without notable input processing deficits were selected, as determined from scores on such tests included in the database. Multiple logistic regression analyses on the individual trial data then revealed that, overall, the greater the target word's frequency, the smaller the chance of a nonword error. Critically, the frequency regression coefficients were equally large in naming and repetition, thus supporting the view that repetition is fully lexically mediated.

As this example application demonstrates, this database has proven to be a powerful tool for testing computational models (e.g., Dell et al., 2007; Nozari et al., 2010) and extending existing theories of language processing and language impairments (e.g., Kittredge et al., 2008; Mirman, 2011) and we hope that making it public will accelerate further development of our understanding of the neural basis of language and other cognitive functions.

\section{Acknowledgments}

We are grateful for a long-running grant from the NIH (RO1DC000191) to M.F.S. that supported data collection and the development of the database. Database development was also assisted by support from the Moss Rehabilitation Research Institute. The authors owe a special debt of gratitude to all of the patients who completed these tests (with invariable patience and good cheer), the speech-language pathologists who generously took the time to refer these individuals to us, and the research assistants who conducted most of the testing and coding of the results.

\section{References}

Bozeat S, Lambon Ralph MA, Patterson K, Garrard P, Hodges JR. Non-verbal semantic impairment in semantic dementia. Neuropsychologia. 2000; 38:1207-1215. [PubMed: 10865096]

Breedin SD, Saffran EM. Sentence processing in the face of semantic loss: A case study. Journal of Experimental Psychology: General. 1999; 128(4):547-562. [PubMed: 10650585]

Buxbaum LJ, Saffran EM. Knowledge of object manipulation and object function: Dissociations in apraxic and non-apraxic subjects. Brain and Language. 2002; 82:179-199. [PubMed: 12096875]

Dabul, BL. Apraxia Battery for Adults. Second Edition (ABA-2). PRO-ED; Austin, TX: 2000.

Dell GS, Lawler EN, Harris HD, Gordon JK. Models of errors of omission in aphasic naming. Cognitive Neuropsychology. 2004; 21(2-4):125-145. [PubMed: 21038196]

Dell GS, Martin N, Schwartz MF. A case-series test of the interactive two-step model of lexical access: Predicting word repetition from picture naming. Journal of Memory and Language. 2007; 56(4):490-520. [PubMed: 21085621]

Dell GS, O'Seaghdha PG. Mediated and convergent lexical priming in language production: A comment on Levelt et al (1991). Psychological Review. 1991; 98(4):604-614. [PubMed: 1961775]

Dell GS, Schwartz MF, Martin N, Saffran EM, Gagnon DA. Lexical access in aphasic and nonaphasic speakers. Psychological Review. 1997; 104(4):801-838. [PubMed: 9337631]

Dunn, LM.; Dunn, LM. Examiner's Manual for the PPVT-III: Peabody Picture Vocabulary Test. Third Edition. American Guidance Service; Circle Pines, MN: 1997. 
Foygel D, Dell GS. Models of impaired lexical access in speech production. Journal of Memory and Language. 2000; 43(2):182-216.

Francis, WN.; Kucera, H. Frequency analysis of English usage: Lexicon and grammar. Houghton, Mifflin Co.; Boston: 1982.

Freedman ML, Martin RC. Dissociable components of short-term memory and their relation to longterm learning. Cognitive Neuropsychology. 2001; 18(3):193-226. [PubMed: 20945211]

Freedman ML, Martin RC, Biegler K. Semantic relatedness effects in conjoined noun phrase production: Implications for the role of short-term memory. Cognitive Neuropsychology. 2004; 21(2-4):245-265. [PubMed: 21038203]

Fridriksson J, Moser D, Bonilha L, Morrow-Odom KL, Shaw H, Fridriksson A, et al. Neural correlates of phonological and semantic-based anomia treatment in aphasia. Neuropsychologia. 2007; 45(8): 1812-1822. [PubMed: 17292928]

Howard, D.; Patterson, K. Pyramids and Palm Trees: A test of semantic access from pictures and words. Thames Valley Test Company; Bury St. Edmunds, Suffolk: 1992.

Jokel R, Rochon E, Leonard C. Testing predictions of the interactive activation model in recovery from aphasia after treatment. Brain and Cognition. 2004; 54(3):251-253. [PubMed: 15050786]

Kaplan, E.; Goodglass, H. Boston Naming Test. Lea \& Febiger; Philadelphia, PA: 1983a.

Kaplan, E.; Goodglass, H. 2nd ed.. Lea \& Febiger; Malvern, PA: 1983b. The Assessment of Aphasia and Related Disorders.

Kay, J.; Lesser, R.; Coltheart, M. PALPA: Psycholinguistic Assessments of Language Processing in Aphasia. Lawrence Erlbaum Associates Ltd.; Hove, UK: 1992.

Kertesz, A. Western Aphasia Battery. Grune \& Stratton; New York: 1982.

Kittredge AK, Dell GS, Verkuilen J, Schwartz MF. Where is the effect of frequency in word production? Insights from aphasic picture-naming errors. Cognitive Neuropsychology. 2008; 25(4):463-492. [PubMed: 18704797]

Leonard C, Rochon E, Laird L. Treating naming impairments in aphasia: Findings from a phonological components analysis treatment. Aphasiology. 2008; 22(9):923-947.

Martin N, Ayala J. Measurements of auditory-verbal STM span in aphasia: Effects of item, task, and lexical impairment. Brain and Language. 2004; 89(3):464-483. [PubMed: 15120538]

Martin N, Dell GS. Common mechanisms underlying perseverative and non-perseverative sound and word substitutions. Aphasiology. Special Issue: Verbal perseveration. 2007; 21(10-11):10021017.

Martin N, Schwartz MF, Kohen FP. Assessment of the ability to process semantic and phonological aspects of words in aphasia: A multi-measurement approach. Aphasiology. 2006; 20(2/3/4):154166.

Martin RC, Shelton JR, Yaffee LS. Language processing and working memory: Neuropsychological evidence for separate phonological and semantic capacities. Journal of Memory and Language. 1994; 33:83-111.

Mirman D. Effects of near and distant semantic neighbors on word production. Cognitive, Affective, \& Behavioral Neuroscience. 2011

Nozari N, Kittredge AK, Dell GS, Schwartz MF. Naming and repetition in aphasia: Steps, routes, and frequency effects. Journal of Memory and Language. 2010; 63:541-559. [PubMed: 21076661]

Patterson K, Plaut DC. "Shallow draughts intoxicate the brain": Lessons from cognitive science for cognitive neuropsychology. Topics in Cognitive Science. 2009; 1:39-58.

Roach A, Schwartz MF, Martin N, Grewal RS, Brecher A. The Philadelphia Naming Test: Scoring and rationale. Clinical Aphasiology. 1996; 24:121-133.

Saffran EM, Coslett HB, Keener MT. Differences in word associations to pictures and words. Neuropsychologia. 2003; 41:1541-1546. [PubMed: 12849772]

Saffran EM, Coslett HB, Martin N, Boronat CB. Access to knowledge from pictures but not words in a patient with progressive fluent aphasia. Language and Cognitive Processes. 2003; 18(5/6):725757.

Cogn Neuropsychol. Author manuscript; available in PMC 2012 June 30. 
Schwartz MF, Brecher A. A model-driven analysis of severity, response characteristics, and partial recovery in aphasics' picture naming. Brain and Language. 2000; 73(1):62-91. [PubMed: 10872638]

Schwartz MF, Brecher AR, Whyte J, Klein MG. A patient registry for cognitive rehabilitation research: A strategy for balancing patients' privacy rights with researchers' need for access. Archives of Physical Medicine and Rehabilitation. 2005; 86(9):1807-1814. [PubMed: 16181947]

Schwartz MF, Dell GS. Case series investigations in cognitive neuropsychology. Under review. 2010

Schwartz MF, Dell GS, Martin N, Gahl S, Sobel P. A case-series test of the interactive two-step model of lexical access: Evidence from picture naming. Journal of Memory and Language. 2006; 54(2): 228-264. 
Table 1

Additional tests included in database. Numbers in parentheses indicate the number of patients that completed each test.

\begin{tabular}{|c|c|}
\hline Aphasia diagnostic tests & $\begin{array}{l}\text { Western Aphasia Battery }(\mathrm{N}=104) \\
\text { - } \quad \text { Aphasia Quotient } \\
\text { - } \quad \text { Fluency } \\
\text { - } \\
\text { Comprehension }\end{array}$ \\
\hline & $\begin{array}{l}\text { Boston Diagnostic Aphasia Examination }(\mathrm{N}=106) \\
\text { Boston Naming Test }(\mathrm{N}=104) \\
\text { Dabul Apraxia of Speech Battery }(\mathrm{N}=81)\end{array}$ \\
\hline Speech perception and spoken word recognition tests & $\begin{array}{l}\text { Phoneme discrimination }(\mathrm{N}=148) \\
\text { - } \quad \text { No delay } \\
\text { - } \quad 5 \text { sec delay } \\
\text { Rhyme discrimination }(\mathrm{N}=72) \\
\text { Lexical decision }(\mathrm{N}=147) \\
\text { - } \quad \text { Words } \\
\text { - } \quad \text { Nonwords } \\
\text { O } \quad \text { Overall d' }\end{array}$ \\
\hline Semantic tests & $\begin{array}{l}\text { PNT Picture Name Verification Test }(\mathrm{N}=130) \\
\text { Synonymy triplets } \\
\begin{array}{cc}\text { - } & \text { Nouns }(\mathrm{N}=132) \\
\text { - } & \text { Verbs }(\mathrm{N}=132) \\
\text { - } & \text { Total }(\mathrm{N}=135)\end{array}\end{array}$ \\
\hline & $\begin{array}{l}\text { Peabody Picture Vocabulary Test }(\mathrm{N}=77) \\
\text { Semantic category discrimination }(\mathrm{N}=72) \\
\text { Camel and Cactus Test }(\mathrm{N}=72) \\
\text { Pyramids and Palm Trees Test }(\mathrm{N}=117)\end{array}$ \\
\hline Other cognitive function tests & $\begin{array}{l}\text { Nonword repetition } \\
\begin{array}{l}\text { - } \\
\text { - }\end{array} \quad \text { Version } 1(\mathrm{~N}=50) \\
\text { Short-Term } \text { Memory 2: PNT-based }(\mathrm{N}=72) \\
\text { - } \quad \text { Immediate Serial Recall Span for Words }(\mathrm{N}=74) \\
\text { - } \quad \text { Semantic-Category Probe Test (Semantic STM) }(\mathrm{N}=72) \\
\text { - } \quad \text { Rhyme Probe Test (Phonological STM) }(\mathrm{N}=72) \\
\text { Sentence comprehension (N=115) } \\
\text { - } \quad \text { Lexical foils } \\
\text { - } \quad \text { Reversible foils }\end{array}$ \\
\hline
\end{tabular}


Table 2

Brief descriptions of additional tests included in the database. Full descriptions are provided at http://www.mappd.org/.

\author{
Aphasia diagnostic tests \\ Western Aphasia Battery (Kertesz, 1982) \\ - Aphasia Quotient: Overall severity of language impairment based on weighted composite of 10 \\ WAB subtests. Maximum score: 100. \\ - $\quad$ Fluency: Verbal fluency exhibited by the patient based on informativeness and grammaticality of \\ spontaneous speech when answering questions and describing pictures. Maximum score: 10 . \\ - Comprehension: Patient's performance on yes-no questions, auditory word recognition, and \\ sequential commands. Maximum score: 10. \\ Boston Diagnostic Aphasia Examination (Kaplan \& Goodglass, 1983b) \\ - Value: Severity rating that ranges from " 0 " (neither useful comprehension nor speech output is \\ possible) to " 5 " (normal comprehension and speech output). \\ Boston Naming Test (Kaplan \& Goodglass, 1983a) \\ - A 60 -item picture-naming test with items rank-ordered in terms of their ability to be named. Value: \\ Percent correct \\ Dabul (2000) Apraxia of Speech Battery \\ - A combination of 6 subtests (diadochokinetic rate, increasing word length, limb and oral apraxia, \\ latency and utterance time for polysyllabic words, repetition, and an inventory of articulation \\ characteristics of apraxia) to assess apraxia of speech in adult patients and severity of disorder. \\ Subjects with diagnosed verbal apraxia were afforded a one-sound off leniency in scoring for PNT \\ and PRT errors. Value: severity (mild, moderate, or severe) \\ Speech perception and \\ spoken word recognition \\ tests \\ Phoneme discrimination (Martin, Schwartz, \& Kohen, 2006) \\ - On each trial, the subject hears two recorded words $(n=20)$ or non-words $(n=20)$ and is required to \\ indicate whether the two are the "same" or "different". Non-identical pairs differ by a single onset or \\ final phoneme. Value: Percent correct on 40 trials.
}

Rhyme discrimination (based on Freedman \& R. Martin, 2001)

- On each trial, the subject listens to two words and indicates whether they rhyme by saying or pointing to "Yes" or "No". Value: Percent correct on 30 trials.

Lexical decision

- Auditory lexical decision subtest of the Psycholinguistic Assessment of Language Processing in Aphasia (PALPA) (Kay, Lesser, \& Coltheart, 1992). The 80 real word items, balanced for imageability and frequency, interspersed with 80 pronounceable nonwords. Values: Percent correct acceptance of words, correct rejection of nonwords, and overall $d$ '.

PNT Picture Name Verification Test (Dell, Martin, \& Schwartz, 2007)

- On each trial, the subject must indicate whether the presented picture and spoken name match. The pictures are drawn from 162 of the 175 PNT items. The name is the target, a semantically close foil, a semantically distant foil, a phonologically close nonword foil, or a phonologically distant nonword foil. Testing was conducted in the three separate sessions with items pseudo-randomly assigned to condition and rotated through all conditions across sessions. Value: Precent correct.

Synonymy triplets: Synonymy Judgments with Nouns and Verbs Test (Martin et al., 2006)

- On each trial, the subject views three written words that are spoken aloud by the examiner and must decide which two are most similar in meaning. Half the trials involve nouns (e.g., violin, fiddle, clarinet), the other half verbs (e.g., to repair, to design, to fix). Values: Percent correct for 15 Noun trials, 15 Verb trials, and overall.

Peabody Picture Vocabulary Test (Dunn \& Dunn, 1997) 
- $\quad$ An untimed, norm-referenced vocabulary test arranged in order of increasing difficulty and representing various parts of speech. On each trial, a heard word must be matched to one of four pictures that best represents its meaning. Value: Standard score on the 204-item PPVT.

Semantic category discrimination (based on Freedman \& R. Martin, 2001)

- On each trial, the subject listens to two words and indicates whether they are members of the same semantic category by saying or pointing to "Yes" or "No". Value: Percent correct on 40 trials.

Camel and Cactus Test (Bozeat et al., 2000)

- $\quad$ On each trial, a pictured item must be matched to the closest associate among a set of four pictured choices (e.g., wine matched to: grapes, cherry, strawberry, orange). Value: Percent correct on 64 trials.

Pyramids and Palm Trees Test (Howard \& Patterson, 1992)

- On each trial, a pictured item must be matched to the closest associate among a set of two pictured choices (e.g., fish matched to: $\underline{\text { cat }}$, dog). Value: Percent correct on 52 trials.

Other cognitive function tests
Nonword repetition

- Version 1 (Dell, Martin, \& Schwartz, 2007): Each of 60 nonwords was created by changing one consonant and one vowel in 60 concrete one- and two-syllable words to create a phonologically legal nonword that was clearly different from the word. Nonword targets were presented individually on tape for the subject to repeat interspersed with the words from which they were derived (filler trials). Value: Percent correct on 60 trials.

- Version 2: Each of 60 nonwords was created by pseudo-randomly changing two phonemes in a PNT target word to create a pronounceable nonword that was clearly different from the word. Nonword targets were presented individually on tape for the subject to repeat. Value: Percent correct on 60 trials.

Short-Term Memory

- Immediate Serial Recall Span for Words (R. Martin, Shelton, \& Yaffee, 1994): Subjects were required to repeat 10 lists of one-syllable words, starting with two-word lists ("wine-dream") and increasing up to five-word lists, if possible ("soul-fear-art-dream-shoe"). Value: Span length (X.Y, where $\mathrm{X}$ is longest list that with at least $50 \%$ correct recall and $\mathrm{Y}$ is the proportion correct on the next list out of $50 \%$ ).

- $\quad$ Semantic-Category Probe Test (Freedman \& R. Martin, 2001): The subject listens to a list of three or more words and must determine if the final word is from the same category as any of the preceding words by saying or pointing to "Yes" or "No". The list of words gradually increases and the test is terminated when the subject drops below 75\% correct. Value: Maximum list length with 75\% or higher accuracy.

- $\quad$ Rhyme Probe Test (Freedman \& R. Martin, 2001): The subject listens to a list of three or more words and must determine is the final word rhymes with any of the preceding words by saying or pointing to "Yes" or "No". The list of words gradually increases and the test is terminated when the subject drops below $75 \%$ correct. Value: Maximum list length with $75 \%$ or higher accuracy.

Sentence comprehension (Breedin \& Saffran, 1999)

- On each trial, the subject must indicate which of two pictures matches the spoken sentence. The distractor pictures are either "reversible" (patient and agent reversed) or "lexical" (action or character differs from the sentence). The test has two versions (A and B) consisting of different sentences. Value: Percent correct on 30 items of each type. 